\title{
Backward error analysis for multi-symplectic integration methods
}

\author{
Brian Moore $^{1}$, Sebastian Reich ${ }^{2}$ \\ 1 Department of Mathematics and Statistics, University of Surrey, and Department of \\ Mathematics, Imperial College, London; e-mail: b.moore@ic.ac.uk \\ 2 Department of Mathematics, Imperial College, London; e-mail: s.reich@ic.ac.uk
}

Received October 25, 2001 / Revised version received July 22, 2002 /

Published online - (C) Springer-Verlag 2003

\begin{abstract}
Summary. A useful method for understanding discretization error in the numerical solution of ODEs is to compare the system of ODEs with the modified equations obtained through backward error analysis, and using symplectic integration for Hamiltonian ODEs provides more incite into the modified equations. In this paper, the ideas of symplectic integration are extended to Hamiltonian PDEs, and this paves the way for the development of a local modified equation analysis solely as a useful diagnostic tool for the study of these types of discretizations. In particular, local conservation laws of energy and momentum are not preserved exactly when symplectic integrators are used to discretize, but the modified equations are used to derive modified conservation laws that are preserved to higher order along the numerical solution. These results are also applied to the nonlinear wave equation.
\end{abstract}

Mathematics Subject Classification (1991):

\section{Introduction}

A method known as backward error analysis (BEA), in which a system of differential equations is compared with the modified equations that are satisfied by the numerical solution, is one of the best ways to analyze the effects of discretization error in a numerical solution. The modified equations are found by truncating an asymptotic expansion about the approximated solution. Currently, there are many results concerning the BEA of numerical methods for ODEs. In particular, the modified equations of a Hamiltonian system are also

Correspondence to: $\mathrm{S}$. Reich

\begin{tabular}{|c|c|c|c|c|c|}
\hline & 21 & 045 & & & $\mathrm{Co}$ \\
\hline & Jour. No & Ms. No. & & $\begin{array}{l}\text { Disk Received } \\
\text { Disk Used } \square\end{array}$ & $\begin{array}{l}\text { Corrupted } \square \\
\text { Mismatch } \square\end{array}$ \\
\hline
\end{tabular}


Hamiltonian if and only if the integrator used is symplectic, meaning the numerical method preserves the symplectic structure of the ODE, and these types of integrators have proved to give accurate and efficient results for the long-time numerical integration of these systems (cf. $[1,6,12,19])$. There are fewer results concerning BEA in relation to PDEs, but the idea of a modified PDE has been presented as a means of determining the accuracy and stability of numerical integrators for PDEs (cf. [24]).

The idea of symplectic integration has more recently been extended to the multi-symplectic structure of Hamiltonian PDEs in a first paper by Marsden et al. [15], who use the multi-symplectic structure of wave equations. Their approach derives a numerical scheme from the Lagrangian formulation in firstorder field theory using a discrete variational principle. We use an approach based on the multi-symplectic structure, which was suggested by Bridges and Reich [5]. This approach uses the application of a symplectic method to each independent variable, and defines multi-symplectic integrators as methods that preserve a discrete version of the symplectic conservation law (CL). We make a first step toward understanding multi-symplectic integrators by way of BEA. In particular, we formally derive modified equations that will be used as a diagnostic tool to verify energy and momentum conservation.

The following model problem is used throughout this text. Assuming the spatial domain $[0, l]$ and the time domain $\left[t^{0}, t^{F}\right]$, consider the Lagrangian functional

$$
\mathcal{L}=\int_{t^{0}}^{t^{F}} \int_{0}^{l} L \mathrm{~d} x \mathrm{~d} t
$$

with the Lagrangian density

$$
L=L\left(u_{t}, u_{x}, u\right)=\frac{1}{2} u_{t}^{2}-\sigma\left(u_{x}\right)-f(u),
$$

where $\sigma$ and $f$ are smooth functions, $u=u(x, t)$ and $v=v(x, t)$ for $x, t \in \mathbb{R}$, and $u_{t}=\partial u / \partial t, u_{x}=\partial u / \partial x$. Then, using the variational principle, we derive the nonlinear wave equation

$$
u_{t t}=\partial_{x} \sigma^{\prime}\left(u_{x}\right)-f^{\prime}(u)
$$

from the Euler-Lagrange equation

$$
\partial_{t} L_{u_{t}}+\partial_{x} L_{u_{x}}-L_{u}=0,
$$

where $\partial_{t}$ and $\partial_{x}$ denote partial differentiation with respect to $t$ and $x$ respectively.

Equation (1.2) can also be stated as a system of first-order equations such that

(1.3) $-v_{t}-p_{x}=f^{\prime}(u), \quad u_{t}=v, \quad 0=p+\sigma^{\prime}(w), \quad u_{x}=w$, 
and this is equivalent to a multi-symplectic PDE, which is given in abstract form by

$$
\mathbf{K} z_{t}+\mathbf{L} z_{x}=\nabla_{z} S(z)
$$

where $z$ is the $d$-dimensional vector of state variables, $\mathbf{K}, \mathbf{L} \in \mathbb{R}^{d \times d}$ are constant and skew-symmetric, and $S: \mathbb{R}^{d} \rightarrow \mathbb{R}$ is a smooth function. For the specific case of the nonlinear wave equation we have taken

$$
\mathbf{K}=\left[\begin{array}{cccc}
0 & -1 & 0 & 0 \\
1 & 0 & 0 & 0 \\
0 & 0 & 0 & 0 \\
0 & 0 & 0 & 0
\end{array}\right] \quad \text { and } \quad \mathbf{L}=\left[\begin{array}{cccc}
0 & 0 & 0 & -1 \\
0 & 0 & 0 & 0 \\
0 & 0 & 0 & 0 \\
1 & 0 & 0 & 0
\end{array}\right]
$$

with $z=[u, v, w, p]^{T}$, and

$$
S(z)=\frac{v^{2}}{2}+p w+\sigma(w)+f(u) .
$$

This multi-symplectic formulation is a natural generalization of Hamiltonian ODEs to PDEs. Examples of PDEs that have been formulated this way can be found in [2-4].

Now we are concerned with numerical schemes that conserve a multi-symplectic CL. There is numerical evidence that these schemes locally conserve energy and momentum remarkably well, though not exactly (cf. [20]). Note that local conservation of these properties is a much stronger result than the global conservation achieved in past results (cf. [16]). The multi-symplectic formalism can be used to understand the CLs of energy and momentum for equations of the form (1.4) by way of BEA, and we present an extensive analysis for doing so in the following text. We emphasize that we do not intend in this paper to use BEA to interpret the complete solution behavior in light of the modified equations. Such an undertaking would strongly depend on the PDE as well as its initial and boundary conditions.

The outline of the paper is as follows. First, in $\$ 2$, we discuss the common CLs of symplecticity, energy, and momentum, that are associated with multisymplectic PDEs, and show the existence of these laws for the nonlinear wave equation. In $\S 3$ a multi-symplectic discretization, referred to as the Euler box scheme, is presented. It is shown that a spatial discretization, resulting in a Hamiltonian system of ODEs, implies a spatially discrete energy CL, and the same is shown for the conservation of momentum using a time discretization. After a complete discretization, the CLs of energy and momentum are no longer conserved exactly. However, under sufficient smoothness assumptions, we show that standard BEA can be used to formally derive a modified $\mathrm{CL}$ that is preserved to higher order in one independent variable. These results are derived in $\S 4$. While this approach to BEA has its advantages, a different 
method of BEA can be used to derive modified equations for the PDE and these are used to get CLs that are preserved to higher order in both space and time. This is the subject of $\S 5$. In $\S 6$, we consider numerical simulations that demonstrate our main results, and make concluding remarks in $\$ 7$.

\section{Conservation laws}

To begin, we introduce the CLs of symplecticity, energy, and momentum, that are discussed throughout this text. Each of these CLs can be derived from the equation (1.4), and in the following sections we discuss these laws in light of multi-symplectic integration.

Following [2,3], a multi-symplectic CL

$$
\partial_{t} \omega+\partial_{x} \kappa=0
$$

where

$$
\omega=\mathrm{d} z \wedge \mathbf{K} \mathrm{d} z \quad \text { and } \quad \kappa=\mathrm{d} z \wedge \mathbf{L d} z
$$

is derived directly from (1.4). Here, we use wedge product notation such that $\mathrm{d} z$ denotes the vector of differentials.

This can be simplified by taking a (non-unique) splitting of the matrices $\mathbf{K}$ and $\mathbf{L}$ such that

$$
\mathbf{K}=\mathbf{K}_{+}+\mathbf{K}_{-} \quad \text { and } \quad \mathbf{L}=\mathbf{L}_{+}+\mathbf{L}_{-},
$$

with

$$
\mathbf{K}_{+}^{T}=-\mathbf{K}_{-} \quad \text { and } \quad \mathbf{L}_{+}^{T}=-\mathbf{L}_{-} .
$$

A splitting of this form immediately implies

$$
\mathrm{d} z \wedge \mathbf{K}_{+} \mathrm{d} z=\mathrm{d} z \wedge \mathbf{K}_{-} \mathrm{d} z \quad \text { and } \quad \mathrm{d} z \wedge \mathbf{L}_{+} \mathrm{d} z=\mathrm{d} z \wedge \mathbf{L}_{-} \mathrm{d} z
$$

hence (2.1) holds with

$$
\omega=\mathrm{d} z \wedge \mathbf{K}_{+} \mathrm{d} z \quad \text { and } \quad \kappa=\mathrm{d} z \wedge \mathbf{L}_{+} \mathrm{d} z .
$$

This splitting also becomes helpful as we study a multi-symplectic discretization of (1.4), and this will become evident in the next section.

Similar to the analysis in [2,3], we can also derive the CLs of energy and momentum. Using the time invariance of (1.4), an energy CL can easily be derived by taking the inner product (denoted on $\mathbb{R}^{d}$ by $\langle\cdot, \cdot\rangle$ ) of (1.4) with $z_{t}$. Then

$$
\left\langle z_{t}, \mathbf{L} z_{x}\right\rangle=\left\langle z_{t}, \nabla_{z} S(z)\right\rangle=\partial_{t} S(z),
$$


since the skew-symmetry of $\mathbf{K}$ implies $\left\langle z_{t}, \mathbf{K} z_{t}\right\rangle=0$. Noting that

$$
\begin{aligned}
\left\langle z_{t}, \mathbf{L} z_{x}\right\rangle & =\left\langle z_{t}, \mathbf{L}_{+} z_{x}\right\rangle+\left\langle z_{t}, \mathbf{L}_{-} z_{x}\right\rangle \\
& =\left\langle z_{t}, \mathbf{L}_{+} z_{x}\right\rangle-\left\langle z_{x}, \mathbf{L}_{+} z_{t}\right\rangle \\
& =\partial_{x}\left\langle z_{t}, \mathbf{L}_{+} z\right\rangle-\partial_{t}\left\langle z_{x}, \mathbf{L}_{+} z\right\rangle,
\end{aligned}
$$

we obtain the energy CL

$$
\partial_{t} E+\partial_{x} F=0
$$

where

$$
E=S(z)+\left\langle z_{x}, \mathbf{L}_{+} z\right\rangle \text { and } F=-\left\langle z_{t}, \mathbf{L}_{+} z\right\rangle
$$

are known respectively as the energy density and the energy flux. Similarly, the spatial invariance of (1.4) can be used to take the inner product of (1.4) with $z_{x}$, which gives

$$
\partial_{x} S(z)=\left\langle z_{x}, \mathbf{K} z_{t}\right\rangle=\partial_{t}\left\langle z_{x}, \mathbf{K}_{+} z\right\rangle-\partial_{x}\left\langle z_{t}, \mathbf{K}_{+} z\right\rangle .
$$

Thus, the momentum $\mathrm{CL}$ is given by

$$
\partial_{x} G+\partial_{t} I=0
$$

where

$$
G=S(z)+\left\langle z_{t}, \mathbf{K}_{+} z\right\rangle \quad \text { and } \quad I=-\left\langle z_{x}, \mathbf{K}_{+} z\right\rangle .
$$

Notice that the momentum CL is achieved by reversing the roles of space and time (the inner product is taken with $z_{x}$ rather than $z_{t}$ ), and using the same steps used to get the energy CL. Thus, it is not necessary to derive each CL separately, and in order to avoid this redundancy in the remainder of the text, we shall consider only the energy CL in the general derivations with the understanding that the same holds for momentum, while both will be considered for specific case of the nonlinear wave equation.

For the system (1.3), take a splitting of the matrices $\mathbf{K}$ and $\mathbf{L}$ defined by

$$
\mathbf{K}_{+}=\left[\begin{array}{cccc}
0 & -1 & 0 & 0 \\
0 & 0 & 0 & 0 \\
0 & 0 & 0 & 0 \\
0 & 0 & 0 & 0
\end{array}\right] \quad \text { and } \quad \mathbf{L}_{+}=\left[\begin{array}{cccc}
0 & 0 & 0 & -1 \\
0 & 0 & 0 & 0 \\
0 & 0 & 0 & 0 \\
0 & 0 & 0 & 0
\end{array}\right]
$$

and define $\mathbf{K}_{-}$and $\mathbf{L}_{-}$such that (2.2) is satisfied. Using this splitting, the CL of symplecticity automatically becomes (2.1) for

$$
\omega=\mathrm{d} u \wedge \mathrm{d} v \quad \text { and } \quad \kappa=\mathrm{d} u \wedge \mathrm{d} p .
$$

Using (2.4), it is also easy to show that (2.3) is satisfied with

$$
E=\frac{1}{2} v^{2}+\sigma(w)+f(u)=\frac{1}{2} u_{t}^{2}+\sigma\left(u_{x}\right)+f(u)
$$


and

$$
F=v p=-u_{t} \sigma^{\prime}\left(u_{x}\right) .
$$

Under periodic boundary conditions, conservation of total energy

$$
H(t)=\int_{0}^{l} E(t, x) \mathrm{d} x
$$

holds since

$$
\frac{\mathrm{d}}{\mathrm{d} t} H=\int_{0}^{l} \partial_{t} E \mathrm{~d} x=-\int_{0}^{l} \partial_{x} F \mathrm{~d} x,
$$

but the energy CL is valid independent of the boundary conditions. Similarly, (2.5) holds for

$$
G=\sigma(w)+p w-\frac{1}{2} v^{2}+f(u)=\sigma\left(u_{x}\right)-\sigma^{\prime}\left(u_{x}\right) u_{x}-\frac{1}{2} u_{t}^{2}+f(u)
$$

and

$$
I=w v=u_{x} u_{t} .
$$

\section{Multi-Symplectic discretizations}

Here and throughout the remainder of the paper, we use the notation $z^{n, i}$ to denote a numerical approximation of $z\left(x_{n}, t_{i}\right)$, for $n=0,1,2, \ldots, J$ and $i=0,1,2, \ldots, \tau$, where $J$ is the number of grid points and $\tau$ is the number of time steps. We also define $l / J=\Delta x=x_{n}-x_{n-1}$ and $\left(t^{F}-t^{0}\right) / \tau=\Delta t=$ $t_{i}-t_{i-1}$. Then, using both forward and backward differences, we define a discrete approximation to $z_{x}$ by

$$
\delta_{x}^{+} z^{n}:=\frac{z^{n+1}-z^{n}}{\Delta x} \quad \text { and } \quad \delta_{x}^{-} z^{n}:=\frac{z^{n}-z^{n-1}}{\Delta x},
$$

where $\delta_{x}^{ \pm}$has been introduced for the sake of compact notation. Furthermore, we define the central difference approximation for second order derivatives with respect to $x$ by

$$
\delta_{x}^{2} z^{n}:=\frac{z^{n+1}-2 z^{n}+z^{n-1}}{\Delta x^{2}}=\delta_{x}^{+} \delta_{x}^{-} z^{n}
$$

and the same can be done for discretizations in time.

It was briefly discussed in the introduction that a multi-symplectic PDE given by (1.4) exploits the symplectic structure for each independent variable $x$ and $t$. Now, as we consider a discretization of (1.4), we use a similar idea and apply a symplectic Euler discretization to each independent variable. 
This yields a first-order explicit one-step numerical method that preserves a discrete version of the CL of symplecticity. We refer to this method as the Euler box scheme, given by

$$
\mathbf{K}_{+} \delta_{t}^{+} z^{n, i}+\mathbf{K}_{-} \delta_{t}^{-} z^{n, i}+\mathbf{L}_{+} \delta_{x}^{+} z^{n, i}+\mathbf{L}_{-} \delta_{x}^{-} z^{n, i}=\nabla_{z} S\left(z^{n, i}\right) .
$$

Proposition 1 The Euler box scheme (3.1) satisfies a discrete multi-symplectic conservation law

$$
\delta_{t}^{+} \omega^{n, i}+\delta_{x}^{+} \kappa^{n, i}=0
$$

where

$$
\omega^{n, i}=\mathrm{d} z^{n, i-1} \wedge \mathbf{K}_{+} \mathrm{d} z^{n, i} \quad \text { and } \quad \kappa^{n, i}=\mathrm{d} z^{n-1, i} \wedge \mathbf{L}_{+} \mathrm{d} z^{n, i} .
$$

Proof. Consider the discrete variational equation

$$
\mathbf{K}_{+} \delta_{t}^{+} \mathrm{d} z^{n, i}+\mathbf{K}_{-} \delta_{t}^{-} \mathrm{d} z^{n, i}+\mathbf{L}_{+} \delta_{x}^{+} \mathrm{d} z^{n, i}+\mathbf{L}_{-} \delta_{x}^{-} \mathrm{d} z^{n, i}=S_{z z}\left(z^{n, i}\right) \mathrm{d} z^{n, i} .
$$

Now take the wedge product of this equation with $\mathrm{d} z^{n, i}$, and notice that we have

$$
\mathrm{d} z^{n, i} \wedge S_{z z}\left(z^{n, i}\right) \mathrm{d} z^{n, i}=0,
$$

because $S_{z z}$ is symmetric. Then, for the terms containing $\delta_{t}^{ \pm}$, we get

$$
\begin{aligned}
\mathrm{d} z^{n, i} & \wedge \mathbf{K}_{+} \delta_{t}^{+} \mathrm{d} z^{n, i}+\mathrm{d} z^{n, i} \wedge \mathbf{K}_{-} \delta_{t}^{-} \mathrm{d} z^{n, i} \\
= & \mathrm{d} z^{n, i} \wedge \mathbf{K}_{+} \delta_{t}^{+} \mathrm{d} z^{n, i}+\delta_{t}^{-} \mathrm{d} z^{n, i} \wedge \mathbf{K}_{+} \mathrm{d} z^{n, i} \\
& =\delta_{t}^{+}\left(\mathrm{d} z^{n, i-1} \wedge \mathbf{K}_{+} \mathrm{d} z^{n, i}\right) .
\end{aligned}
$$

Doing the same for the terms containing $\delta_{x}^{ \pm}$yields (3.2).

Example. Discretizing (1.3) first in space gives

$$
-v_{t}^{n}-\delta_{x}^{+} p^{n}=f^{\prime}\left(u^{n}\right), \quad u_{t}^{n}=v^{n}, \quad p^{n}=-\sigma^{\prime}\left(w^{n}\right), \quad \delta_{x}^{-} u^{n}=w^{n},
$$

which is equivalent to

$$
v_{t}^{n}=\delta_{x}^{+} \sigma^{\prime}\left(\delta_{x}^{-} u^{n}\right)-f^{\prime}\left(u^{n}\right), \quad u_{t}^{n}=v^{n} .
$$

Notice that, for $\Delta x=1, \sigma(w)=w^{2} / 2+w^{3} / 3$, and $f^{\prime}(u)=0$, these equations become the Fermi-Pasta-Ulam problem (cf. [9]). With periodic boundary conditions, the system of ordinary differential equations (3.4) is also Hamiltonian in the classical sense where

$$
H=\sum_{n=1}^{J} E^{n}, \quad \text { and } \quad E^{n}=\frac{1}{2}\left(v^{n}\right)^{2}+\sigma\left(\delta_{x}^{-} u^{n}\right)+f\left(u^{n}\right)
$$


is the approximated energy density. Applying a symplectic Euler discretization in time, (3.4) becomes

$$
\delta_{t}^{+} v^{n, i}=\delta_{x}^{+} \sigma^{\prime}\left(\delta_{x}^{-} u^{n, i}\right)-f^{\prime}\left(u^{n, i}\right), \quad \delta_{t}^{-} u^{n, i}=v^{n, i} .
$$

Then a short calculation shows that (3.2) is satisfied with

$$
\omega^{n, i}=\mathrm{d} u^{n, i-1} \wedge \mathrm{d} v^{n, i}, \quad \text { and } \quad \kappa^{n, i}=\mathrm{d} u^{n-1, i} \wedge \mathrm{d} p^{n, i},
$$

such that

$$
\mathrm{d} p^{n, i}=-\sigma^{\prime \prime}\left(w^{n, i}\right) \delta_{x}^{-} \mathrm{d} u^{n, i}
$$

Alternatively, to use the approach based on the Lagrangian formulation in [15], we approximate the Lagrangian functional (1.1) with

$$
\mathcal{L} \approx \sum_{i=1}^{\tau} \sum_{n=1}^{J} L^{n, i},
$$

for the discrete Lagrangian density

$$
L^{n, i}=L\left(\delta_{t}^{-} u^{n, i}, \delta_{x}^{-} u^{n, i}, u^{n, i}\right)=\frac{1}{2}\left(\delta_{t}^{-} u^{n, i}\right)^{2}-\sigma\left(\delta_{x}^{-} u^{n, i}\right)-f\left(u^{n, i}\right) .
$$

Now, using the associated discrete form of the variational principle, we derive a discrete form of the Euler-Lagrange equation given by

$$
\delta_{t}^{+} L_{\delta_{t}^{-} u^{n, i}}+\delta_{x}^{+} L_{\delta_{x}^{-} u^{n, i}}+L_{u^{n, i}}=0,
$$

and this yields the discretization

$$
\delta_{t}^{2} u^{n, i}=\delta_{x}^{+} \sigma^{\prime}\left(\delta_{x}^{-} u^{n, i}\right)-f^{\prime}\left(u^{n, i}\right),
$$

which is equivalent to the Euler box scheme. For a comparison of these two approaches, see [17].

\section{Discrete conservation laws and backward error analysis}

Certain semi-discrete CLs can be found for multi-symplectic equations. Then, using standard BEA, we can formally derive a modified Hamiltonian system of ODEs, which in turn gives modified semi-discrete CLs. 


\subsection{Standard backward error for the wave equation}

Consider the Hamiltonian ODE (3.4) with periodic boundary conditions, and for the sake of simplicity let $\sigma(w)=w^{2} / 2$, which yields the well-known nonlinear Klein-Gordon equation. Then re-scaling the velocities $v^{n}=\hat{v}^{n} / \Delta x$ and discretizing in time by a symplectic Euler method yields the equivalent equations

$$
\begin{aligned}
& \hat{v}^{n, i+1}-\hat{v}^{n, i}=\varepsilon\left(u^{n+1, i}-2 u^{n, i}+u^{n-1, i}\right)-\varepsilon \Delta x^{2} f^{\prime}\left(u^{n, i}\right), \\
& u^{n, i+1}-u^{n, i}=\varepsilon \hat{v}^{n, i+1},
\end{aligned}
$$

where $\varepsilon=\Delta t / \Delta x$. Now if we let $\mathbf{y}^{i}=\left[\mathbf{u}^{i}, \hat{\mathbf{v}}^{i}\right]$, where $\mathbf{u}^{i}$ and $\hat{\mathbf{v}}^{i}$ are defined to be the vectors containing $u^{n, i}$ and $\hat{v}^{n, i}$ respectively for all $n$, then these equations define a map denoted by

$$
\mathbf{y}^{i+1}=\Psi_{\varepsilon}\left(\mathbf{y}^{i}\right) .
$$

This symplectic map is $\varepsilon$-close to the identity. Thus, one can find an approximate Hamiltonian flow map according to the results in $[1,19]$. The difference between these maps can be made exponentially small in terms of the parameter $\varepsilon$ which implies near conservation of total energy over exponentially long time intervals. The validity of this statement depends crucially upon letting $\varepsilon \rightarrow 0$, and this is obviously a much stronger requirement than the usual CFL stability condition $\varepsilon \leq 1$, which often implies excellent conservation of energy even for nonlinear problems.

On the other hand, one could formally apply standard BEA to (3.4) based on the fact that the time discretization is a simple splitting method. This widely used formal method of BEA, which we refer to as BEA-1, is well understood for ODEs, and is described by Sanz-Serna and Calvo [21][pages 129-131]. More specifically, for separable Hamiltonians, such that $H=T+V$ is the sum of potential and kinetic terms, the modified Hamiltonian for the symplectic Euler method is given by

$$
\bar{H}_{\rho}=H+\frac{\Delta t}{2}\{T, V\}+\frac{\Delta t^{2}}{12}(\{T,\{T, V\}\}+\{V,\{V, T\}\})+\ldots,
$$

where $\rho$ is the number of modifications. This formula is obtained using the BCH formula, where $\{\cdot, \cdot\}$ denotes the canonical bracket (cf. [21][Chapter 12]).

A spatial discretization of a Hamiltonian PDE, using a symplectic integrator, yields a Hamiltonian system of ODEs. Thus, BEA-1 can be used to formally derive the modified equations. Choose a splitting of the Hamiltonian (3.5) such that $H=T+V$ with

$$
T=\frac{1}{2} \sum_{n=1}^{J}\left(v^{n}\right)^{2} \quad \text { and } \quad V=\sum_{n=1}^{J} \frac{\left(\delta_{x}^{-} u^{n}\right)^{2}}{2}+f\left(u^{n}\right) .
$$


This implies, for example,

$$
\{T, V\}=\sum_{n=1}^{J}\left(v^{n} \delta_{x}^{2} u^{n}-v^{n} f^{\prime}\left(u^{n}\right)\right) .
$$

To get a second modification, one would need the additional terms

$$
\{T,\{T, V\}\}=\sum_{n=1}^{J}\left(\left(v^{n}\right)^{2} f^{\prime \prime}\left(u^{n}\right)+v^{n} \delta_{x}^{2} v^{n}\right)
$$

and

$$
\{V,\{V, T\}\}=\sum_{n=1}^{J}\left(\left(\delta_{x}^{2} u^{n}\right)^{2}-\left(f^{\prime}\left(u^{n}\right)\right)^{2}\right) .
$$

Higher order modifications are found in a similar manner.

Upon first observation we see that the modification terms also depend on powers of $1 / \Delta x$, which are all hidden in finite difference approximations. Hence, we must make the following necessary but reasonable assumptions, in order to guarantee that the associated modified Hamiltonian (4.1) indeed depends only on $\Delta t$.

A1: The solutions of the given PDE remain smooth over the time interval of interest.

A2: For $\Delta x$ sufficiently small and $\Delta t$ satisfying a CFL stability condition, all necessary finite difference approximations can be bounded by a constant that does not depend on $\Delta x$ and $\Delta t$ (numerical smoothness).

This implies, for example, the estimate

$$
\left|\delta_{x}^{2} u^{n}\right| \leq K,
$$

for some constant $K$, which implies $\{T, V\}=\mathcal{O}(1)$ and the first modification term is $\mathcal{O}(\Delta t)$. Naturally, $\mathbf{A 1}$ and $\mathbf{A} \mathbf{2}$ imply that the $j$ th modification term in (4.1) is indeed $\mathcal{O}\left(\Delta t^{j}\right)$. Unfortunately, the rigorous proof of $\mathbf{A 2}$ is strongly problem dependent for nonlinear PDEs, and it is a proof beyond the scope of this paper. Clearly, a CFL stability argument is a necessary condition to ensure that $\mathbf{A 2}$ holds, but it is not sufficient. Some nonlinear stability argument is needed. However, these assumptions do allow a formal application of BEA-1 to these types of problems with $\varepsilon=\Delta t$. Hence, we assume A1 and $\mathbf{A 2}$ hold throughout the text. 


\subsection{Semi-discrete conservation laws}

For multi-symplectic equations that have been semi-discretized to yield a system of ODEs, a spatially discrete energy CL is preserved exactly, and similarly a semi-discretization in time leads to a momentum CL that is preserved exactly.

Proposition 2 Applying a symplectic Euler discretization in space to (1.4) yields an exact semi-discrete energy $C L$

$$
\partial_{t} E^{n}+\delta_{x}^{+} F^{n}=0
$$

with

$$
E^{n}=S\left(z^{n}\right)+\left\langle\delta_{x}^{-} z^{n}, \mathbf{L}_{+} z^{n}\right\rangle, \quad \text { and } \quad F^{n}=-\left\langle z_{t}^{n-1}, \mathbf{L}_{+} z^{n}\right\rangle,
$$

and a symplectic Euler discretization in time yields an exact semi-discrete momentum $C L$

$$
\partial_{x} G^{i}+\delta_{t}^{+} I^{i}=0
$$

with

$$
G^{i}=S\left(z^{n}\right)+\left\langle\delta_{t}^{-} z^{i}, \mathbf{K}_{+} z^{i}\right\rangle, \quad \text { and } \quad I^{i}=-\left\langle z_{x}^{i-1}, \mathbf{K}_{+} z^{i}\right\rangle .
$$

Proof. First apply a symplectic Euler discretization to (1.4) in space to get the semi-discrete equation

$$
\mathbf{K} z_{t}^{n}+\mathbf{L}_{+} \delta_{x}^{+} z^{n}+\mathbf{L}_{-} \delta_{x}^{-} z^{n}=\nabla_{z} S\left(z^{n}\right) .
$$

Taking the inner product with $z_{t}^{n}$ yields

$$
\left\langle z_{t}^{n}, \mathbf{L}_{+} \delta_{x}^{+} z^{n}\right\rangle+\left\langle z_{t}^{n}, \mathbf{L}_{-} \delta_{x}^{-} z^{n}\right\rangle=\left\langle z_{t}^{n}, \nabla_{z} S\left(z^{n}\right)\right\rangle=\partial_{t} S\left(z^{n}\right),
$$

then adding and subtracting like terms and using properties of the inner product gives

$$
\begin{aligned}
\partial_{t} S\left(z^{n}\right)+\partial_{t}\left\langle\delta_{x}^{-} z^{n}, \mathbf{L}_{+} z^{n}\right\rangle & =\left\langle z_{t}^{n}, \mathbf{L}_{+} \delta_{x}^{+} z^{n}\right\rangle+\left\langle\delta_{x}^{-} z_{t}^{n}, \mathbf{L}_{+} z^{n}\right\rangle \\
& =\delta_{x}^{+}\left\langle z_{t}^{n-1}, \mathbf{L}_{+} z^{n}\right\rangle .
\end{aligned}
$$

Thus, we have proved (4.3). To continue our formal analysis and find a semidiscrete momentum CL, simply discretize (1.4) in time (leaving space continuous and disregarding stability issues) with a symplectic Euler scheme, then take the inner product with $z_{x}^{i}$ to get (4.5).

We emphasize here that this result is independent of the boundary conditions. 


\subsection{Modified equations and conservation laws}

Returning to the nonlinear wave equation, we consider the semi-discrete CLs and use BEA-1 to get semi-discrete modified CLs. Apply Proposition 2 to the spatially discrete system (3.3) to obtain

$$
\partial_{t} E^{n}=\delta_{x}^{+}\left(v^{n-1} \sigma^{\prime}\left(\delta_{x}^{-} u^{n}\right)\right) .
$$

Therefore, the semi-discrete CL (4.3) is satisfied with the obvious substitution for $F^{n}$.

Applying BEA-1 and using (4.1) with (4.2), implies the modified energy density

$$
\bar{E}_{1}^{n}=\frac{1}{2}\left(\left(v^{n}\right)^{2}+\left(\delta_{x}^{-} u^{n}\right)^{2}+\Delta t\left(v^{n} \delta_{x}^{2} u^{n}-v^{n} f^{\prime}\left(u^{n}\right)\right)\right)+f\left(u^{n}\right) .
$$

In fact, the modified Hamiltonian can be written

$$
\bar{H}_{\rho}=\sum_{n=1}^{J} \bar{E}_{\rho}^{n},
$$

for any number of modifications $\rho$. (Once again we assume periodic boundary conditions, but $\$ 5$ will provide a completely local picture that is independent of boundary conditions.) Thus, the modified Hamiltonian system of ODEs becomes

$$
\begin{aligned}
-v_{t}^{n}+\delta_{x}^{2} u^{n}-\frac{\Delta t}{2} \delta_{x}^{2} v^{n} & =f^{\prime}\left(u^{n}\right)-\frac{\Delta t}{2} f^{\prime \prime}\left(u^{n}\right) v^{n}, \\
u_{t}^{n}-\frac{\Delta t}{2} \delta_{x}^{2} u^{n} & =v^{n}-\frac{\Delta t}{2} f^{\prime}\left(u^{n}\right) .
\end{aligned}
$$

This modified system of equations can also be written in the form of the multi-symplectic PDE (1.4) with $z=[u, v, w, \phi, \psi]^{T}$ and

$$
S=\frac{v^{2}-w^{2}}{2}+f(u)-\frac{\Delta t}{2}\left(f^{\prime}(u) v-\phi \psi\right),
$$

for $w=\psi=u_{x}$ and $\phi=v_{x}$. Now (4.9) can be found using the analog of (4.4) for this modified multi-symplectic PDE.

A higher order semi-discrete modified energy CL

$$
\partial_{t} \bar{E}_{\rho}^{n}+\delta_{x}^{+} \bar{F}_{\rho}^{n}=0
$$

can be found in the obvious way. However, this can become quite cumbersome as higher order derivatives are involved for higher order modifications and the phase space of the modified multi-symplectic PDE becomes very large. Furthermore, finding a canonical way to get a modified multi-symplectic PDE using BEA-1 is not straightforward.

Based on a more classical approach, we can obtain the semi-discrete CLs of energy and momentum using only the modified Hamiltonian (4.7) and its 
corresponding equations of motion. For example, the modified energy density (4.6) can be differentiated with respect to $t$ to give

$$
\begin{aligned}
\partial_{t} \bar{E}_{1}^{n}= & \delta_{x}^{+}\left[\delta_{x}^{-} u^{n}\left(v^{n-1}+\frac{\Delta t}{2}\left(\delta_{x}^{2} u^{n-1}-f^{\prime}\left(u^{n-1}\right)\right)\right)\right. \\
& +\frac{\Delta t}{2} v^{n}\left(\delta_{x}^{-} v^{n}+\frac{\Delta t}{2}\left(\delta_{x}^{-} \delta_{x}^{2} u^{n}-\delta_{x}^{-} f^{\prime}\left(u^{n}\right)\right)\right) \\
& \left.-\frac{\Delta t}{2} \delta_{x}^{-} v^{n}\left(v^{n}+\frac{\Delta t}{2}\left(\delta_{x}^{2} u^{n}-f^{\prime}\left(u^{n}\right)\right)\right)\right] .
\end{aligned}
$$

Then the system of equations (4.8) implies

$$
\partial_{t} \bar{E}_{1}^{n}=\delta_{x}^{+}\left[u_{t}^{n-1} \delta_{x}^{-} u^{n}+\frac{\Delta t}{2}\left(v^{n} \delta_{x}^{-} u_{t}^{n}-u_{t}^{n} \delta_{x}^{-} v^{n}\right)\right],
$$

which is of the form (4.9). This is a very simple and specific case, and the fully general problem is more complex. However, it can be shown that given a modified Hamiltonian (4.1) with any number of modifications using BEA-1, a modified CL can be found using a recursion relation, and a detailed proof of this result is found in [17].

Similar results can be formally derived concerning a momentum CL. We now make use of $\mathbf{A 2}$ with respect to the discrete time derivatives. First notice that the time discrete Klein-Gordon equation can formally be written

$$
w_{x}^{i}=\delta_{t}^{2} u^{i}+f^{\prime}\left(u^{i}\right), \quad u_{x}^{i}=w^{i},
$$

which, with the index $i$ ranging form minus to plus infinity, is also a Hamiltonian system in space where the Hamiltonian is given by

$$
\hat{H}=\sum_{i=-\infty}^{\infty} G^{i}, \quad \text { where } \quad G^{i}=\frac{1}{2}\left(\left(w^{i}\right)^{2}+\left(\delta_{t}^{-} u^{i}\right)^{2}\right)-f\left(u^{i}\right)
$$

We can differentiate the momentum flux given by $G^{i}$ to get

$$
\partial_{x} G^{i}=w^{i} w_{x}^{i}+\delta_{t}^{-} u^{i} \delta_{t}^{-} w^{i}-f^{\prime}\left(u^{i}\right) w^{i}=\delta_{t}^{+}\left(w^{i-1} \delta_{t}^{-} u^{i}\right)
$$

where we have used (4.11), and this is just the semi-discrete momentum CL (4.5).

Consider a splitting of (4.12) such that $\hat{H}=\hat{T}+\hat{V}$ for

$$
\hat{T}=\frac{1}{2} \sum_{i=-\infty}^{\infty}\left(w^{i}\right)^{2} \quad \text { and } \quad \hat{V}=\sum_{i=-\infty}^{\infty} \frac{1}{2}\left(\delta_{t}^{-} u^{i}\right)^{2}-f\left(u^{i}\right) .
$$

Then the first modification can be found by evaluating

$$
\{\hat{T}, \hat{V}\}=\sum_{i=-\infty}^{\infty}\left(w^{i} \delta_{t}^{2} u^{i}+w^{i} f^{\prime}\left(u^{i}\right)\right),
$$


which implies the modified momentum flux

$$
\bar{G}_{1}^{i}=\frac{1}{2}\left(\left(w^{i}\right)^{2}+\left(\delta_{t}^{-} u^{i}\right)^{2}+\Delta x\left(w^{i} \delta_{t}^{2} u^{i}+w^{i} f^{\prime}\left(u^{i}\right)\right)\right)-f\left(u^{i}\right) .
$$

Thus, the modified semi-discrete equations of motion are given by

$$
\begin{aligned}
w_{x}^{i} & =\delta_{t}^{2} u^{i}+f^{\prime}\left(u^{i}\right)-\frac{\Delta x}{2}\left(\delta_{t}^{2} w^{i}+w^{i} f^{\prime \prime}\left(u^{i}\right)\right) \\
u_{x}^{i} & =w^{i}+\frac{\Delta x}{2}\left(\delta_{t}^{2} u^{i}+f^{\prime}\left(u^{i}\right)\right) .
\end{aligned}
$$

Following a similar procedure used to get (4.10), the semi-discrete modified momentum CL is obtained by differentiating $\bar{G}_{1}^{i}$, given in (4.13), with respect to $x$ to get

$$
\begin{aligned}
\partial_{x} \bar{G}_{1}^{i}= & \delta_{t}^{-} u^{i} \delta_{t}^{-} u_{x}^{i}+w^{i} w_{x}^{i}-f^{\prime}\left(u^{i}\right) u_{x}^{i} \\
& +\frac{\Delta x}{2}\left(w_{x}^{i} \delta_{t}^{2} u^{i}+w^{i} \delta_{t}^{2} u_{x}^{i}+w_{x}^{i} f^{\prime}\left(u^{i}\right)+w^{i} f^{\prime \prime}\left(u^{i}\right) u_{x}^{i}\right) .
\end{aligned}
$$

Then substituting

$$
\begin{aligned}
w^{i} w_{x}^{i}= & u_{x}^{i} \delta_{t}^{2} u^{i}+f^{\prime}\left(u^{i}\right) u_{x}^{i} \\
& -\frac{\Delta x}{2}\left(u_{x}^{i} \delta_{t}^{2} w^{i}+w_{x}^{i} \delta_{t}^{2} u^{i}+w^{i} f^{\prime \prime}\left(u^{i}\right) u_{x}^{i}+w_{x}^{i} f^{\prime}\left(u^{i}\right)\right),
\end{aligned}
$$

gives

$$
\begin{aligned}
\partial_{x} \bar{G}_{1}^{i} & =\delta_{t}^{-} u^{i} \delta_{t}^{-} u_{x}^{i}+u_{x}^{i} \delta_{t}^{2} u^{i}+\frac{\Delta x}{2}\left(w^{i} \delta_{t}^{2} u_{x}^{i}-u_{x}^{i} \delta_{t}^{2} w^{i}\right) \\
& =\delta_{t}^{+}\left(u_{x}^{i-1} \delta_{t}^{-} u^{i}\right)+\frac{\Delta x}{2} \delta_{t}^{+}\left(w^{i} \delta_{t}^{-} u_{x}^{i}-u_{x}^{i} \delta_{t}^{-} w^{i}\right)
\end{aligned}
$$

and this is a semi-discrete modified momentum CL.

It is important to notice that there are two sets of modified equations here, depending on how we do the BEA. In fact, the modified equations here are only for the systems of ODEs that result from a semi-discretization of the PDE. The advantage is that we can obtain semi-discrete CLs, but the price paid for these is an incomplete picture of the modified equations for the PDE. However, this problem is addressed in $§ 5$.

\subsection{Numerical verification of discrete conservation laws}

Conservation of total energy can be monitored by checking that the Hamiltonian converges to a constant value as $\Delta t \rightarrow 0$. For the semi-discrete energy density $\bar{E}_{\rho}^{n}$, conservation of total energy can be checked directly because the modification terms contain no time derivatives, meaning no additional 
discretization error is introduced. Thus, the discrete Hamiltonian $\bar{H}_{\rho}^{i}$, which is just the Hamiltonian evaluated at the numerical solution that is obtained using the symplectic Euler scheme, satisfies

$$
\bar{H}_{\rho}^{i}=\bar{H}_{\rho}\left(t_{i}\right)+\mathcal{O}\left(\Delta t^{\rho+1}\right)
$$

where $\bar{H}_{\rho}\left(t_{i}\right)$ is the semi-discrete Hamiltonian given in (4.7) evaluated along the exact solution of the modified equation.

In contrast, the local energy CL does contain time derivatives, and in order to maintain the order of convergence of the modified CL, we must use an approximation of the appropriate order for each time derivative. In general, we can derive a semi-discrete energy CL (4.9) for any number of modifications $\rho$, and we know that this conservation law is satisfied along the numerical solution up to an $\mathcal{O}\left(\Delta t^{\rho+1}\right)$ error. Hence, to check the order of convergence numerically, this CL must be discretized using no less than an $\mathcal{O}\left(\Delta t^{\rho+1}\right)$ method. As we consider the fully discrete CL, let

$$
\delta_{t}^{(\rho+1)} z^{n, i}=\partial_{t} z^{n}\left(t_{i}\right)+\mathcal{O}\left(\Delta t^{\rho+1}\right)
$$

be any discretization of order $\rho+1$. Then, we have the residual

$$
r_{e}^{n, i}=\delta_{t}^{(\rho+1)} \bar{E}_{\rho}^{n, i}+\delta_{x}^{+} \bar{F}_{\rho}^{n, i}=\mathcal{O}\left(\Delta t^{\rho+1}\right)
$$

provided each time derivative of $\bar{F}_{\rho}^{n}$ is also discretized to the appropriate order. These higher order discretizations can be achieved in many ways, but one of the simplest and most practical is to use symmetric differencing (cf. [8][page 16]). The same analysis holds for discrete momentum CLs, where the spatial derivatives in (4.5) with $\rho$ modifications must be approximated by an $\mathcal{O}\left(\Delta x^{\rho+1}\right)$ method.

For the nonlinear wave equation, (4.14) is immediately satisfied for both the modified and unmodified equations. Moreover, (4.15) with $\rho=0$ and $\rho=1$ is satisfied for

$$
F^{n, i}=-\left(\delta_{t}^{(1)} u^{n-1, i}\right) \delta_{x}^{-} u^{n, i}
$$

and

$$
\bar{F}_{1}^{n, i}=-\left(\delta_{t}^{(2)} u^{n-1, i}\right) \delta_{x}^{-} u^{n, i}-\frac{\Delta t}{2}\left(v^{n, i} \delta_{t}^{(1)} \delta_{x}^{-} u^{n, i}-\left(\delta_{t}^{(1)} u^{n, i}\right) \delta_{x}^{-} v^{n, i}\right),
$$

respectively, where one can use, for example, $\delta_{x}^{(1)}=\delta_{x}^{+}$and $\delta_{t}^{(2)}=\left(\delta_{t}^{+}+\right.$ $\left.\delta_{t}^{-}\right) / 2$.

\section{Revised backward error analysis}

A second method of BEA, which we refer to as the revised method or BEA-2 , can also be considered, and though it may be somewhat less practical for ODEs, it becomes more useful in the context of PDEs. 


\subsection{Backward error analysis for ODEs}

First consider the Hamiltonian ODE

$$
\mathbf{J} y_{t}=\nabla_{y} H(y), \quad \mathbf{J}=\left[\begin{array}{cc}
0 & -1 \\
1 & 0
\end{array}\right],
$$

for $y \in \mathbb{R}^{2}$, and the symplectic Euler scheme

$$
\mathbf{J}_{+} \delta_{t}^{+} y^{i}+\mathbf{J}_{-} \delta_{t}^{-} y^{i}=\nabla_{y} H\left(y^{i}\right) .
$$

Under the stated smoothness assumptions, a standard Taylor series expansion of an interpolating function $y(t)$ gives

$$
\delta_{t}^{+} y\left(t_{i}\right)=y_{t}\left(t_{i}\right)+\frac{\Delta t}{2} y_{t t}\left(t_{i}\right)+\frac{\Delta t^{2}}{6} y_{t t t}\left(t_{i}\right)+\ldots,
$$

and we also have

$$
\delta_{t}^{-} y\left(t_{i}\right)=y_{t}\left(t_{i}\right)-\frac{\Delta t}{2} y_{t t}\left(t_{i}\right)+\frac{\Delta t^{2}}{6} y_{t t t}\left(t_{i}\right)-\ldots .
$$

Thus, we can replace $\delta_{t}^{+} y^{i}$ and $\delta_{t}^{-} y^{i}$ by (5.2) and (5.3) respectively and obtain the modified system of equations

$$
\mathbf{J} y_{t}+\frac{\Delta t}{2} \mathbf{A} y_{t t}=\nabla_{y} H(y),
$$

where $\mathbf{A}=\left(\mathbf{J}_{+}-\mathbf{J}_{-}\right)$is symmetric. This is a first modification because we have ignored all terms of order higher than $\Delta t$. In general, we obtain the modified system of equations

$$
\mathbf{J} y_{t}+\frac{\Delta t}{2} \mathbf{A} y_{t t}+\frac{\Delta t^{2}}{6} \mathbf{J} y_{t t t}+\ldots+\frac{\Delta t^{\rho}}{(\rho+1) !} \mathbf{S}_{\rho} \partial_{t}^{\rho+1} y=\nabla_{y} H(y)
$$

for $\rho$ modifications, where

$$
\mathbf{S}_{\rho}=\left\{\begin{array}{ll}
\mathbf{J}, & \forall \rho \text { even } \\
\mathbf{A}, & \forall \rho \text { odd }
\end{array} .\right.
$$

Note that the equation obtained by differentiation of (5.1) with respect to $t$ can be used to recursively eliminate the higher order derivatives, and yield the modified equations obtained from BEA-1, but this becomes increasingly more complicated for higher order modifications.

Now take the inner product of the modified equations (5.5) with $y_{t}$ to obtain

$$
\begin{aligned}
0=\left\langle y_{t}, \mathbf{J} y_{t}\right\rangle & =\left\langle y_{t}, \nabla_{y} H(y)\right\rangle-\frac{\Delta t}{2}\left\langle y_{t}, \mathbf{A} y_{t t}\right\rangle-\ldots-\frac{\Delta t^{\rho}}{(\rho+1) !}\left\langle y_{t}, \mathbf{S}_{\rho} \partial_{t}^{\rho+1} y\right\rangle \\
& =\frac{\mathrm{d}}{\mathrm{d} t} \tilde{H}_{\rho}\left(y, y_{t}, \ldots, \partial_{t}^{\rho} y\right),
\end{aligned}
$$


where

$$
\tilde{H}_{\rho}=H(y)-\frac{\Delta t}{4}\left\langle y_{t}, \mathbf{A} y_{t}\right\rangle-\frac{\Delta t^{2}}{6}\left\langle y_{t}, \mathbf{J} y_{t t}\right\rangle+\ldots
$$

is now a conserved quantity. In fact, the modified equations (5.5) can be written as a Hamiltonian ODE over an enlarged phase space with Hamiltonian function $\tilde{H}_{\rho}$. This is the subject of the following.

Theorem 1 The modified system of equations (5.5) with $\rho$ modifications is equivalent to the Hamiltonian system

$$
\tilde{\mathbf{J}} \tilde{y}_{t}=\nabla_{\tilde{y}} \tilde{H}_{\rho}(\tilde{y}) .
$$

Here, $\tilde{y}$ stands for

$$
\tilde{y}=\tilde{y}_{\rho}=\left[y_{0}, y_{1}, y_{2}, \ldots, y_{\rho}\right]^{T} \quad \text { where } \quad y_{k}=\left[y_{k}^{(1)}, y_{k}^{(2)}, \ldots, y_{k}^{(k)}\right]^{T},
$$

with $y_{0}=y$ and $y_{k}^{(j)}=\partial_{t}^{j} y$ for all $k=1,2, \ldots, \rho$. Defining

$$
k_{-}=\left\{\begin{array}{ll}
\frac{k}{2}, & \forall k \text { even } \\
\frac{k-1}{2}, & \forall k \text { odd }
\end{array} \quad \text { and } \quad k_{+}=\left\{\begin{array}{ll}
\frac{k}{2}, & \forall k \text { even } \\
\frac{k+1}{2}, & \forall k \text { odd }
\end{array},\right.\right.
$$

we have

$$
\tilde{H}_{\rho}=H+\sum_{k=1}^{\rho}(-1)^{k} \frac{\Delta t^{k}}{(k+1) !}\left[\sum_{j=1}^{k_{+}} \alpha(-1)^{j+1}\left(y_{k}^{(k+1-j)}\right)^{T} \mathbf{S}_{k} y_{k}^{(j)}\right]
$$

with

$$
\alpha=\left\{\begin{array}{ll}
\frac{1}{2}, & \text { for } j=(k+1) / 2 \\
1, & \text { otherwise }
\end{array} .\right.
$$

In addition, let $J_{i, j} \in \mathbb{R}^{2 \times 2}$ denote the matrix elements of the block matrix $\tilde{\mathbf{J}}=\tilde{\mathbf{J}}_{\rho}$, such that

$$
J_{1, k_{\Sigma}}=-J_{k_{\Sigma}-(k-1), k_{\Sigma}-1}=\ldots=(-1)^{k_{-}} J_{k_{\Sigma}-k_{+}, k_{\Sigma}-k_{-}}=\frac{\Delta t^{k}}{(k+1) !} \mathbf{S}_{k},
$$

where

$$
k_{\Sigma}=1+\sum_{j=1}^{k} j, \quad \text { for } \quad k=1,2, \ldots, \rho,
$$

and since $\tilde{\mathbf{J}}$ is skew-symmetric we only need to list the diagonal and upper triangular nonzero elements for any given $k$. 
Proof. In the case $\rho=1$, we have

$$
\tilde{H}_{1}(\tilde{y})=H(y)-\frac{\Delta t}{4}\left(y_{1}^{(1)}\right)^{T} \mathbf{A} y_{1}^{(1)}=H(y)-\frac{\Delta t}{4} y_{t}^{T} \mathbf{A} y_{t}
$$

and

$$
\tilde{y}=\left[\begin{array}{c}
y \\
y_{1}^{(1)}
\end{array}\right]=\left[\begin{array}{c}
y \\
y_{t}
\end{array}\right], \quad \tilde{\mathbf{J}}=\left[\begin{array}{cc}
\mathbf{J} & \frac{\Delta t}{2} \mathbf{A} \\
\frac{-\Delta t}{2} \mathbf{A} & \mathbf{0}
\end{array}\right]
$$

which is clearly equivalent to (5.4). Note, the elements of $\tilde{\mathbf{J}}$ are given by

$$
J_{1,1}=\mathbf{J} \quad \text { and } \quad J_{1,2}=-J_{2,1}=\frac{\Delta t}{2} \mathbf{A}
$$

with all remaining elements zero.

The phase space becomes more enlarged with each modification, and the modified system for $\rho$ modifications is built upon the modified system for $\rho-1$ modifications. Assume the theorem holds for $\rho=m$, and consider the system (5.6) for $\rho=m+1$. Clearly, the modified Hamiltonian can be written

$$
\tilde{H}_{m+1}=\tilde{H}_{m}+H_{m+1},
$$

and we can write

$$
\tilde{\mathbf{J}}_{m+1}\left(\tilde{y}_{m+1}\right)_{t}=\left[\begin{array}{cc}
\tilde{\mathbf{J}}_{m} & \mathbf{B} \\
-\mathbf{B}^{T} & \mathbf{J}_{m+1}
\end{array}\right]\left[\begin{array}{c}
\tilde{y}_{m} \\
y_{m+1}
\end{array}\right]_{t}=\nabla \tilde{H}_{m+1},
$$

where

$$
\begin{gathered}
\tilde{\mathbf{J}}_{m} \in \mathbb{R}^{2 m_{\Sigma} \times 2 m_{\Sigma}}, \quad \mathbf{B} \in \mathbb{R}^{2 m_{\Sigma} \times 2(m+1)}, \quad \mathbf{J}_{m+1} \in \mathbb{R}^{2(m+1) \times 2(m+1)}, \\
\tilde{y}_{m} \in \mathbb{R}^{2 m_{\Sigma}}, \quad \text { and } \quad y_{m+1} \in \mathbb{R}^{2(m+1)} .
\end{gathered}
$$

Replacing $k$ by $m+1$ in (5.8) shows that the only nonzero element of $\mathbf{B}$ is

$$
B_{1,(m+1)}=J_{1,(m+1)_{\Sigma}}=\frac{\Delta t^{m+1}}{(m+2) !} \mathbf{S}_{m+1},
$$

and the remaining terms in (5.8) make up the matrix $\mathbf{J}_{m+1}$. Thus, the equation

$$
\tilde{\mathbf{J}}_{m}\left(\tilde{y}_{m}\right)_{t}+\mathbf{B}\left(y_{m+1}\right)_{t}=\nabla \tilde{H}_{m}
$$

is just

$$
\mathbf{J} y_{t}+\frac{\Delta t}{2} \mathbf{A} y_{t t}+\frac{\Delta t^{2}}{6} \mathbf{J} y_{t t t}+\ldots+\frac{\Delta t^{m+1}}{(m+2) !} \mathbf{S}_{m+1} \partial_{t} y_{m+1}^{(m+1)}=\nabla_{y} H(y) .
$$

Now the equation

$$
-\mathbf{B}^{T}\left(\tilde{y}_{m}\right)_{t}+\mathbf{J}_{m+1}\left(y_{m+1}\right)_{t}=\nabla H_{m+1}
$$

is used successively to give the additional equations

$$
\partial_{t} y_{0}=y_{m+1}^{(1)}, \quad \text { and } \quad \partial_{t} y_{m+1}^{(i)}=y_{m+1}^{(i+1)} \quad \text { for } \quad i=1,2, \ldots, m,
$$

which implies (5.5), and by induction this proves the theorem. 
This modified Hamiltonian $\tilde{H}_{\rho}$ is equivalent to the modified Hamiltonian derived using standard BEA up to $\mathcal{O}\left(\Delta t^{\rho+1}\right)$. The only difference here is that the modification terms contain explicit time derivatives, and this has implications for checking the conservation of total energy numerically. To make this clear, notice that the discrete modified Hamiltonian must satisfy

$$
\tilde{H}_{\rho}^{i}=\tilde{H}_{\rho}\left(t_{i}\right)+\mathcal{O}\left(\Delta t^{\rho+1}\right),
$$

similar to (4.14). Thus, it is a necessary condition that each time derivative of $\tilde{E}_{\rho}$ is approximated to the appropriate order to ensure the desired order of convergence, and this is easily done using symmetric differencing (cf. [8][page 16]).

Notice also that the modified equations are equivalent to an Euler-Lagrange equation which is derived from the appropriate Lagrangian. For example, if $\rho=1$ the modified equations can be derived from the Lagrangian density

$$
\tilde{L}_{1}=H(y)+\frac{1}{2} y_{t}^{T} \mathbf{J} y+\frac{\Delta t}{4} y_{t}^{T} \mathbf{A} y_{t} .
$$

In general, the modified Lagrangian can be written

$$
\begin{aligned}
\tilde{L}_{\rho}= & H(y)+\sum_{j=0}^{\rho_{-}}(-1)^{j} \frac{\Delta t^{2 j}}{2(2 j+1) !}\left(\partial_{t}^{j+1} y\right)^{T} \mathbf{J} \partial_{t}^{j} y \\
& +\sum_{j=1}^{\rho_{+}}(-1)^{j-1} \frac{\Delta t^{2 j-1}}{2(2 j) !}\left(\partial_{t}^{j} y\right)^{T} \mathbf{A} \partial_{t}^{j} y,
\end{aligned}
$$

where $\rho_{-}$and $\rho_{+}$are defined by replacing $k$ with $\rho$ in (5.7).

We strictly consider the ODE case here, but we can apply these ideas to Hamiltonian PDEs in the following way. First, we can use the semi-discretized Hamiltonian system of PDEs then apply the revised BEA to the resulting system of ODEs to obtain a modified Hamiltonian system similar to (5.6). We refer to this method as BEA-2, and note that the results are similar to the application of BEA-1. However, these ideas can be applied to PDEs in a more complete manner.

\subsection{Backward error analysis for PDEs}

The revised BEA can also be applied to a multi-symplectic PDE in both space and time, and we call this BEA-3. Now consider (3.1). Using (5.2)-(5.3), one can derive the modified PDE

$$
\mathbf{K} z_{t}+\frac{\Delta t}{2}\left(\mathbf{K}_{+}-\mathbf{K}_{-}\right) z_{t t}+\mathbf{L} z_{x}+\frac{\Delta x}{2}\left(\mathbf{L}_{+}-\mathbf{L}_{-}\right) z_{x x}=\nabla_{z} S(z)
$$


which can also be written as a multi-symplectic PDE. First let

$$
\mathbf{M}=\left(\mathbf{K}_{+}-\mathbf{K}_{-}\right), \quad \text { and } \quad \mathbf{N}=\left(\mathbf{L}_{+}-\mathbf{L}_{-}\right),
$$

and note that these are both symmetric. Then for $r=z_{t}$ and $s=z_{x}$, we have

$$
\mathbf{K} z_{t}+\frac{\Delta t}{2} \mathbf{M} r_{t}+\mathbf{L} z_{x}+\frac{\Delta x}{2} \mathbf{N} s_{x}=\nabla_{z} S(z),
$$

but this is equivalent to

$$
\tilde{\mathbf{K}} \tilde{z}_{t}+\tilde{\mathbf{L}} \tilde{z}_{x}=\nabla_{\tilde{z}} \tilde{S}_{\rho}(\tilde{z}),
$$

where $\tilde{z}=[z, r, s]^{T}$,

$$
\tilde{\mathbf{K}}=\left[\begin{array}{ccc}
\mathbf{K} & \frac{\Delta t}{2} \mathbf{M} & \mathbf{0} \\
\frac{-\Delta t}{2} \mathbf{M} & \mathbf{0} & \mathbf{0} \\
\mathbf{0} & \mathbf{0} & \mathbf{0}
\end{array}\right], \quad \tilde{\mathbf{L}}=\left[\begin{array}{ccc}
\mathbf{L} & \mathbf{0} & \frac{\Delta x}{2} \mathbf{N} \\
\mathbf{0} & \mathbf{0} & \mathbf{0} \\
\frac{-\Delta x}{2} \mathbf{N} & \mathbf{0} & \mathbf{0}
\end{array}\right]
$$

and

$$
\tilde{S}_{1}(\tilde{z})=S-\frac{\Delta t}{2} r^{T} \mathbf{M} r-\frac{\Delta x}{2} s^{T} \mathbf{N} s .
$$

Higher order modifications are found in the same way as the ODE case, and the general formulation for $\rho$ modifications is derived in the obvious way.

A modified local energy CL can be derived from this new multi-symplectic formulation or, more directly, by taking the inner product of (5.9) with $z_{t}$ and rearranging terms. This approach is completely independent of the boundary conditions because it provides us with a modified energy density $\tilde{E}$ and a modified energy flux $\tilde{F}$ without referring to a globally defined Hamiltonian. Now, evaluation of this conservation law along the numerical solution is accomplished using

$$
r_{e}^{n, i}=\delta_{t}^{(\rho+1)} \tilde{E}_{\rho}^{n, i}+\delta_{x}^{(\rho+1)} \tilde{F}_{\rho}^{n, i}=\mathcal{O}\left(\Delta x^{\rho+1}+\Delta t^{\rho+1}\right),
$$

where each derivative of both $\tilde{E}_{\rho}$ and $\tilde{F}_{\rho}$ is approximated to the appropriate order. 
The modified equations in this case can also be obtained from a Lagrangian formulation. In fact, we have

$$
\begin{aligned}
\tilde{L}_{\rho}=S(z) & +\sum_{j=0}^{\rho_{-}}(-1)^{j} \frac{\Delta t^{2 j}}{2(2 j+1) !}\left(\partial_{t}^{j+1} z\right)^{T} \mathbf{K} \partial_{t}^{j} z \\
& +\sum_{j=1}^{\rho_{+}}(-1)^{j-1} \frac{\Delta t^{2 j-1}}{2(2 j) !}\left(\partial_{t}^{j} z\right)^{T} \mathbf{M} \partial_{t}^{j} z \\
& +\sum_{j=0}^{\rho_{-}}(-1)^{j} \frac{\Delta x^{2 j}}{2(2 j+1) !}\left(\partial_{x}^{j+1} z\right)^{T} \mathbf{L} \partial_{x}^{j} z \\
& +\sum_{j=1}^{\rho_{+}}(-1)^{j-1} \frac{\Delta x^{2 j-1}}{2(2 j) !}\left(\partial_{x}^{j} z\right)^{T} \mathbf{N} \partial_{x}^{j} z
\end{aligned}
$$

for any number of modifications $\rho$, and this implies a variational principle which leads to higher order field theories.

\subsection{The nonlinear wave equation}

First we concern ourselves with the ODE case and discuss conservation of total energy using the Hamiltonian ODEs that result from a semi-discretization of the nonlinear wave equation. After applying BEA-2 to (3.4), the modified equations of motion become

$$
v_{t}^{n}+\frac{\Delta t}{2} v_{t t}^{n}=\delta_{x}^{+} \sigma^{\prime}\left(\delta_{x}^{-} u^{n}\right)-f^{\prime}\left(u^{n}\right), \quad u_{t}^{n}-\frac{\Delta t}{2} u_{t t}^{n}=v^{n},
$$

and this implies the modified energy density

$$
\bar{E}_{1}^{n}=\frac{\left(v^{n}\right)^{2}}{2}+\sigma\left(\delta_{x}^{-} u^{n}\right)+f\left(u^{n}\right)+\frac{\Delta t}{2} u_{t}^{n} v_{t}^{n},
$$

for $\rho=1$. To show that the associated total energy $\bar{H}_{1}$ given in (4.7) is conserved, we take the time derivative of $\bar{E}_{1}^{n}$. Since the equations (5.12) imply

$$
\partial_{t} \bar{E}_{1}^{n}=u_{t}^{n} \delta_{x}^{+} \sigma^{\prime}\left(\delta_{x}^{-} u^{n}\right)+\sigma^{\prime}\left(\delta_{x}^{-} u^{n}\right) \delta_{x}^{-} u_{t}^{n}=\delta_{x}^{+}\left(u_{t}^{n-1} \sigma^{\prime}\left(\delta_{x}^{-} u^{n}\right)\right)
$$

the total energy is clearly conserved under the assumed periodic boundary conditions. It becomes apparent from (5.13) that we have a semi-discrete local energy $C L$ in this case because the BEA has been performed on a system of ODEs rather than the original PDE. Similar results hold for a momentum CL. 
Applying BEA-3 with $\rho=1$, the modified equations of motion are equivalent to the system of PDEs

$$
\begin{aligned}
v_{t}+\frac{\Delta t}{2} v_{t t}+p_{x}+\frac{\Delta x}{2} p_{x x} & =-f^{\prime}(u), \\
u_{x}-\frac{\Delta x}{2} u_{x x} & =w, \\
u_{t}-\frac{\Delta t}{2} u_{t t} & =v, \\
-\sigma^{\prime}(w) & =p .
\end{aligned}
$$

Using the energy density

$$
\tilde{E}_{1}=\frac{v^{2}}{2}+\sigma(w)+f(u)+\frac{\Delta t}{2} u_{t} v_{t}
$$

these equations imply

$$
\partial_{t} \tilde{E}_{1}=-u_{t} p_{x}-p u_{t x}+\frac{\Delta x}{2}\left(p u_{t x x}-u_{t} p_{x x}\right),
$$

because

$$
v v_{t}=-u_{t} p_{x}-f^{\prime}(u) u_{t}-\frac{\Delta t}{2}\left(v_{t t} u_{t}+v_{t} u_{t t}\right)-\frac{\Delta x}{2} u_{t} p_{x x},
$$

and

$$
\sigma^{\prime}(w) w_{t}=-p u_{t x}+\frac{\Delta x}{2} p u_{t x x} .
$$

Therefore, the modified energy flux is

$$
\tilde{F}_{1}=u_{t} p+\frac{\Delta x}{2}\left(u_{t} p_{x}-p u_{t x}\right) .
$$

After discretizing the modified CL we get (5.11) for $\rho=1$ with

$$
\tilde{E}_{1}^{n, i}=\frac{\left(v^{n, i}\right)^{2}}{2}+\sigma\left(w^{n, i}\right)+f\left(u^{n, i}\right)+\frac{\Delta t}{2} \delta_{t}^{(1)} u^{n, i} \delta_{t}^{(1)} v^{n, i},
$$

and

$$
\tilde{F}_{1}^{n, i}=p^{n, i} \delta_{t}^{(2)} u^{n-1, i}+\frac{\Delta x}{2}\left(\delta_{t}^{(2)} u^{n+1, i} \delta_{x}^{(1)} p^{n, i}-p^{n, i} \delta_{t}^{(2)} \delta_{x}^{(1)} u^{n, i}\right) .
$$

Once again we use, for example, $\delta_{t}^{(1)}=\delta_{t}^{+}, \delta_{t}^{(2)}=\left(\delta_{t}^{+}+\delta_{t}^{-}\right) / 2$, etc., to maintain the order of convergence. 


\section{Numerical results}

To check the preservation of the modified CL to higher order numerically, consider a specific case of (1.2), with $\sigma(w)=w^{2} / 2$, and $f(u)=1-\cos (u)$ which gives the sine-Gordon equation

$$
u_{t t}=u_{x x}-\sin (u) .
$$

For all simulations we use the periodic boundary conditions $u\left(x_{0}, t_{i}\right)=$ $u\left(x_{J}, t_{i}\right)$ for $x_{0}=0$ and $x_{J}=1$, and the initial conditions are given by a standard Gaussian with zero velocity, though similar results hold for different initial and boundary conditions.

We use the Euler box scheme to discretize (6.1), then solve the system of equations

$$
\begin{aligned}
\mathbf{v}^{i+1} & =\mathbf{v}^{i}-\Delta t\left(\boldsymbol{\delta}_{x}^{+} \mathbf{w}^{i}+\sin \left(\mathbf{u}^{i}\right)\right) \\
\mathbf{u}^{i+1} & =\mathbf{u}^{i}+\Delta t \mathbf{v}^{i+1} \\
\mathbf{w}^{i+1} & =-\boldsymbol{\delta}_{x}^{-} \mathbf{u}^{i+1}
\end{aligned}
$$

where $\mathbf{u}^{i}=\mathbf{u}\left(t_{i}\right)$ is the vector whose entries are the values of $u$ at each of the grid points with analogous definitions for $\mathbf{v}$ and $\mathbf{w}$, and $\boldsymbol{\delta}_{x}^{ \pm}$represents the matrix obtained by applying $\delta_{x}^{ \pm}$to each entry of a given vector. Using this notation, we solve for the vectors $\mathbf{u}, \mathbf{v}$, and $\mathbf{w}$ at each time step, then use this to evaluate the residual $r_{e}^{n, i}$ given in (4.15). This in turn yields a residual vector $\mathbf{r}_{e}^{n, i}$, which contains the values of $r_{e}^{n, i}$ at each grid point and is used to compute

$$
\max _{n, i}\left|\mathbf{r}_{e}^{n, i}\right|=r_{e}
$$

for each value of $\Delta t$. This procedure is then repeated for different step sizes $\Delta t$, in order to check the order of convergence for modified and unmodified energy CLs. The same is also done for the modified momentum CL where we evaluate $r_{m}$ for $\Delta t$ fixed, while we vary $\Delta x$. All simulations were performed using MATLAB, which made it possible to take advantage of the matrix-vector operations inherent in the problem for this discretization, and we integrate over one period for each simulation.

Using log-log scale, Fig. 6.1 $a$ plots $r_{e}$ as a function of $\Delta t$. with $\Delta x=1 / 40$ and $\Delta t=1 / 40,1 / 80,1 / 160,1 / 400,1 / 600,1 / 1000$, and 1/1500. Similarly, Fig. $6.1 b$ plots $r_{m}$ as a function of $\Delta x$ for the momentum CL. In this case, backward error analysis in space requires that we keep $\Delta t$ fixed while $\Delta x \rightarrow 0$. However, the condition $\Delta t \leq \Delta x$ must be satisfied in order to ensure the stability of the Euler scheme. Therefore, we set $\Delta t=1 / 1500$ for $\Delta x=1 / 40$, $1 / 80,1 / 160,1 / 400,1 / 600,1 / 1000$, and $1 / 1500$. The figure clearly shows that the modified energy CLs, obtained using BEA-1, are conserved to higher 

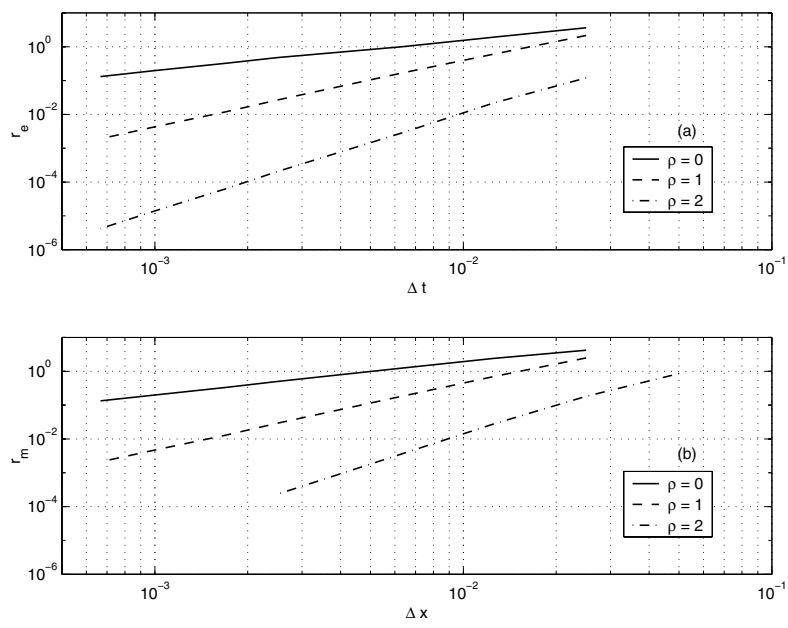

Fig. 6.1. In log-log scale (a) $r_{e}$ as a function of $\Delta t$ and (b) $r^{m}$ as a function of $\Delta x$ where BEA-1 has been used to get the modified equations

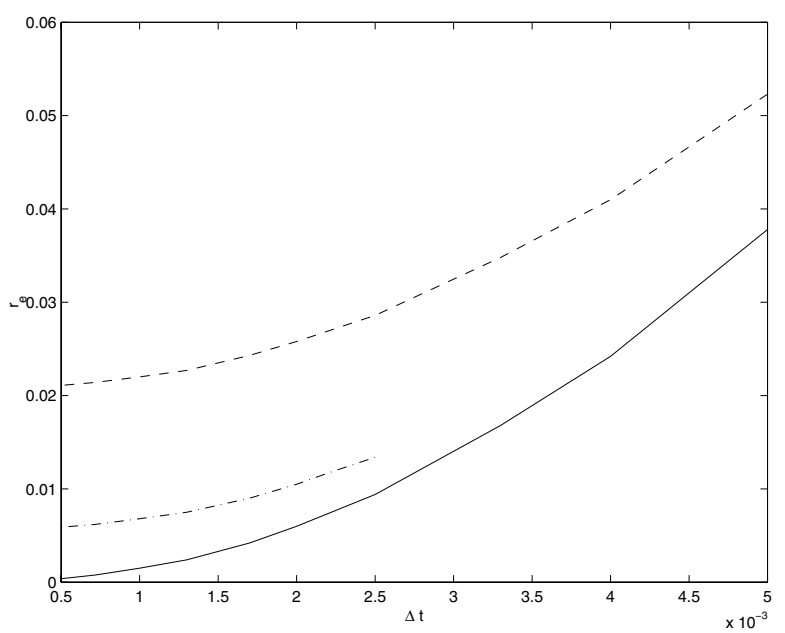

Fig. 6.2. $r_{e}$ as a function of $\Delta t$, for the following curves: BEA-2 (-), and BEA-3 with $\Delta x=.005(--)$ and $\Delta x=.0025(-\cdot-)$

order. For $\rho=0$ the residual converges linearly for both energy and momentum CLs, while $\rho=1$ gives second order convergence and $\rho=2$ gives third order convergence.

Now consider the modified energy CL where the modified equations have been derived using the revised BEA. Here we consider only the energy CL because the stability restriction $\Delta t \leq \Delta x$ makes it difficult to analyze the results for the momentum CL. Hence, each plot here gives $r_{e}$ as a function 


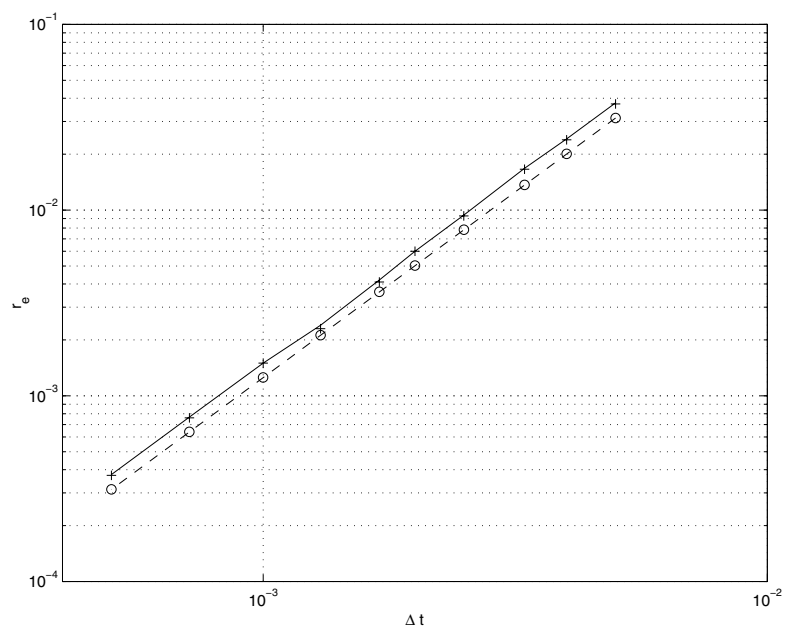

Fig. 6.3. $r_{e}$ as a function of $\Delta t$ in log-log scale for the following curves: BEA-1 (+), BEA-2 (-), and the shifted residual using BEA-3 with $\Delta x=.005$ (- -) and $\Delta x=.01$ (०)

of $\Delta t$, but similar results also hold for the momentum CL. Unless stated otherwise, we set $\Delta x=1 / 100$ with $\Delta t=1 / 200,1 / 250,1 / 300,1 / 400,1 / 500$, 1/600, 1/800, 1/1000, 1/1400, 1/2000. Fig. 6.2 shows a clear difference between BEA-2 and BEA-3. (Note that, due to the stability restriction $\Delta t \leq \Delta x$, this plot can only be plotted for $\Delta x=1 / 400$ with $\Delta t=1 / 400,1 / 500, \ldots$, 1/2000.) For BEA-2, $r_{e} \rightarrow 0$ as $\Delta t \rightarrow 0$, but for BEA-3, $r_{e} \rightarrow C \Delta x^{2}$, for some constant $C$, as $\Delta t \rightarrow 0$. However, as $\Delta x \rightarrow 0$, we have $r_{e} \rightarrow 0$. This is made more clear in the following table, where we see that for $\Delta t \approx 0$, the residual converges to zero like $\Delta x^{2}$, i.e. $r_{e}=\mathcal{O}\left(\Delta t^{2}+\Delta x^{2}\right)$.

\begin{tabular}{|l||c|c|c|c|}
\hline$\Delta x$ & .02 & .01 & .005 & .0025 \\
\hline \hline $\begin{array}{l}\text { Approx. } \\
\lim _{\Delta t \rightarrow 0} r_{e}\end{array}$ & .3184 & .0827 & .0211 & .0059 \\
\hline
\end{tabular}

Using log-log scale, Fig. 6.3 compares each method of BEA. It shows that there is little, if any, difference between BEA-1 and BEA-2. If the $\Delta x^{2}$ dependence of the residual for BEA-3 is neglected, i.e. the parabolae of Fig. 6.2 are shifted to intercept the $y$-axis at zero, then we see that the residual is slightly smaller, and this is true regardless of our choice for $\Delta x$. Overall, BEA-3 gives a better understanding of the error due to discretization of a Hamiltonian PDE. 


\section{Concluding remarks}

The analysis of this paper is specific to the Euler box scheme, but similar results hold using other symplectic methods. For example, an application of the implicit midpoint scheme to each independent variable of (1.4) yields the Preissman box scheme

$$
\mathbf{K} \delta_{t}^{+} z^{n+1 / 2, i}+\mathbf{L} \delta_{x}^{+} z^{n, i+1 / 2}=\nabla_{z} S\left(z^{n+1 / 2, i+1 / 2}\right),
$$

which is discussed in a multi-symplectic setting in [5]. Using Taylor series expansions about $t_{i+1 / 2}$ we find that

$$
\delta_{t}^{+} z\left(t_{i}\right)=z_{t}\left(t_{i+1 / 2}\right)+\frac{\Delta t^{2}}{24} z_{t t t}\left(t_{i+1 / 2}\right)+\mathcal{O}\left(\Delta t^{4}\right),
$$

which implies that the implicit midpoint scheme is a second order method. Hence, the first modification is second order and the modified equations become

$$
\mathbf{K} z_{t}+\Delta t^{2} \mathbf{M} z_{t t t}+\mathbf{L} z_{x}+\Delta x^{2} \mathbf{N} z_{x x x}=\nabla_{z} S(z)
$$

where

$$
\mathbf{M}=\frac{1}{24} \mathbf{K} \quad \text { and } \quad \mathbf{N}=\frac{1}{24} \mathbf{L} .
$$

Now, this can also be written in the form (5.10) for $\tilde{z}=[z, p, q, r, s]^{T}$, and

$$
\tilde{S}_{1}=S+\Delta t^{2} q^{T} \mathbf{M} p+\Delta x^{2} s^{T} \mathbf{N} r
$$

with

$$
\tilde{\mathbf{K}}=\left[\begin{array}{cccccc}
\mathbf{K} & \mathbf{0} & \Delta t^{2} \mathbf{M} & \mathbf{0} & 0 \\
\mathbf{0} & \Delta t^{2} \mathbf{M} & \mathbf{0} & \mathbf{0} & \mathbf{0} \\
\Delta t^{2} \mathbf{M} & \mathbf{0} & \mathbf{0} & \mathbf{0} & \mathbf{0} \\
\mathbf{0} & \mathbf{0} & \mathbf{0} & \mathbf{0} & \mathbf{0} \\
\mathbf{0} & \mathbf{0} & \mathbf{0} & \mathbf{0} & \mathbf{0}
\end{array}\right], \tilde{\mathbf{L}}=\left[\begin{array}{ccccc}
\mathbf{L} & \mathbf{0} & \mathbf{0} & \mathbf{0} & \Delta x^{2} \mathbf{N} \\
\mathbf{0} & \mathbf{0} & \mathbf{0} & \mathbf{0} & \mathbf{0} \\
\mathbf{0} & \mathbf{0} & \mathbf{0} & \mathbf{0} & \mathbf{0} \\
\mathbf{0} & \mathbf{0} & 0 & \Delta x^{2} \mathbf{N} & \mathbf{0} \\
\Delta x^{2} \mathbf{N} & \mathbf{0} & \mathbf{0} & \mathbf{0} & \mathbf{0}
\end{array}\right]
$$

where $p=z_{t}, q=p_{t}, r=z_{x}$, and $s=r_{x}$. Then the modified CLs are easily found.

It is important to notice in this case that the modified CL with one modification is preserved to forth order in $\Delta t$ and $\Delta x$. Just as for the Euler box scheme, the numerical verification of this CL can only be achieved by approximating derivatives to the appropriate order, and the residual of the discrete modified energy $\mathrm{CL}$ with $\rho$ modifications generally satisfies

$$
r_{e}^{n, i}=\mathcal{O}\left(\Delta x^{2 \rho}+\Delta t^{2 \rho}\right) \text {. }
$$


Clearly, this has not been an exhaustive study but only an introduction. One major question that remains concerns using these results to derive estimates for the accuracy of numerical solutions. A first step in this direction is to consider soliton solutions and search for a modified soliton that is satisfied by the modified equations [18], or that better represents the numerical solution [7]. Then additional questions to consider concern the stability of such solitons [4]. Further questions to consider are those related to a theoretical and numerical juxtaposition of multi-symplectic schemes with non-symplectic energy/momentum conserving schemes (cf. [10,11,13,14,20,22,23]), or with other non-conservative schemes.

Throughout this text we have discussed the multi-symplectic integration of Hamiltonian PDEs with application to the nonlinear wave equation. In particular, we considered the modified equations obtained though various methods of backward error analysis, and applied the results to the conservation laws of energy and momentum. It was shown that a semi-discretization of the PDE, which gave a system of ODEs, could be used to derive semidiscrete conservation laws, and performing a formal backward error analysis on this system of ODEs gave modified equations that could be used to get semi-discrete conservation laws. Yet this approach has a shortcoming in that the modified equations did not represent the PDE but the system of ODEs. Hence, an alternative method of BEA was introduced in which a modified multi-symplectic PDE could be derived and used to obtain conservation laws that were preserved to higher order in both space and time.

\section{References}

1. Benettin, G., Giorgilli, A.: On the Hamiltonian interpolation of near to the identity symplectic mappings with application to symplectic integration algorithms. J. Statist. Phys. 74, 1117-1143 (1994)

2. Bridges, T.J.: A geometric formulation of the conservation of wave action and its implications for signature and the classification of instabilities. Proc. R. Soc. London A 453, 1365-1395 (1997)

3. Bridges, T.J.: Multi-symplectic structures and wave propagation. Mathematical Proceedings of the Cambridge Philosophical Society, 121, 147-190 (1997)

4. Bridges, T.J., Derks, G.: Unstable eigenvalues and the linearization about solitary waves and fronts with symmetry. Proc. R. Soc. London A 455, 2427-2469 (1999)

5. Bridges, T.J., Reich, S.: Multi-symplectic integrators: Numerical schemes for Hamiltonian PDEs that conserve symplecticity. Physics Letters A 284, 184-193 (2001)

6. Deuflhard, P., Hermans, J., Leimkuhler, B., Mark, A.E., Reich, S., Skeel, R.D. (eds.): Computational Molecular Dynamics: Challenges Methods Ideas. In Lecture Notes in Computational Science and Engineering Vol. 4, Springer-Verlag, 1999

7. Duran, A., Sanz-Serna, J.M.: The numerical integration of relative equilibrium solutions. The nonlinear Schrødinger equation. IMA J. Numer. Anal. 20, 235-261 (2000)

8. Fornberg, B.: A Practical Guide to Pseudospectral Methods. Cambridge University Press, 1998 
9. Frieseke, G., Pego, R.L.: Solitary waves on FPU lattices: qualitative properties renormalization and continuum limit. Nonlinearity 12, 1601-1628 (1999)

10. Gonzalez, O.: Time integration and discrete Hamiltonian systems. J. Nonlinear Sci. 6, 449-467 (1996)

11. Gonzalez, O., Simo, J.C.: On the stability of symplectic and energy-momentum algorithms for nonlinear Hamiltonian systems with symmetry. Computer Methods in Applied Mechanics and Engineering 134, 197-222 (1996)

12. Hairer, E., Lubich, C.: The life-span of backward error analysis for numerical integrators. Numer. Math. 76, 441-462 (1997)

13. Jiménez, S.: Derivation of the discrete conservation laws for a family of finite difference schemes. Applied Mathematical Computation 64, 13 (1994)

14. Li, S., Vu-Quoc, L.: Finite difference calculus invariant structure of a class of algorithms for the nonlinear Klein-Gordon equation. SIAM J. Numer. Anal. 32(6), 1839-1875 (1995)

15. Marsden, J.E., Patrick, G.P., Shkoller, S.: Multi-symplectic geometry variational integrators and nonlinear PDEs. Commun. Math. Phys. 199(2), 351-395 (1998)

16. McLachlan, R.I.: Symplectic integration of Hamiltonian wave equations. Numer. Math. 66, 465-492 (1994)

17. Moore, B.E.: A modified equations approach for multi-symplectic integration methods. University of Surrey, 2003, In preparation

18. Moore, B.E., Reich, S.: Multi-symplectic integration methods for Hamiltonian PDEs. Future Generation Computer Systems, to appear

19. Reich, S.: Backward error analysis for numerical integrators. SIAM J Numer Anal 36, 1549-1570 (1999)

20. Reich, S.: Multi-symplectic Runge-Kutta collocation methods for Hamiltonian wave equations. J Comp Phys 157, 473-499 (2000)

21. Sanz-Serna, J.M., Calvo, M.P.: Numerical Hamiltonian Problems. London: Chapman and Hall, 1994

22. Strauss, W., Vázquez, L.: Numerical solution of a nonlinear Klein-Gordon equation. J. Comp. Phys. 28, 271-278 (1978)

23. Vu-Quoc, L., Li, S.: Invariant-conserving finite difference algorithms for the nonlinear Klein-Gordon equation. Comput. Meth. Appl. Mech. Engg. 107, 341-391 (1993)

24. Warming, R.F., Hyett, B.J.: The modified equation approach to the stability and accuracy analysis of finite-difference methods. J. Comput. Phys. 14, 159-179 (1974) 